\title{
A New Condition Monitoring Method for Wind Turbines Based on Power Curve Model
}

\author{
*Jianlou Lou ${ }^{1}$, Kai Shan ${ }^{1}$ and Jia $\mathrm{Xu}^{2}$ \\ ${ }^{1}$ School of Information Engineering, Northeast DianLi University, \\ Jilin 132012, China; \\ ${ }^{2}$ Long Yuan(Beijing) Wind Power Engineering Technology CO.,LTD. \\ Beijing 100034, China \\ ${ }^{1}$ loujianlou@qq.com, ${ }^{2} x u j i a @$ clypg.com.cn
}

\begin{abstract}
A new data-driven condition monitoring method for wind turbines is proposed to prevent a turbine failure in a wind farm. The method works through the power curve model which is built with historical SCADA data. New constraints that established based on Betz' law and RC model are developed for governing the power curve model. Since abnormal data has a strong impact on the power curve model, the Inner-DBSCAN algorithm is proposed to reject it. In addition, we use an edge recognition method for normal data to form the power curve model. Then, the turbine operation condition can be monitored through the model. Its effectiveness through industrial studies is confirmed, and the time cost of building the power curve model is only 1.08s.
\end{abstract}

Keywords: wind turbine; condition monitoring; power curve model

\section{Introduction}

Wind power is one of the most promising renewable energy sources. China's newly installed wind turbines capacity reached $23 \mathrm{GW}$ in 2014 and total installed capacity was close to $114 \mathrm{GW}$ by the end of 2014. GWEC predicts that global wind power' rapid development will continue in the next five years. However, due to harsh weather conditions' variations (severe cold, intense heat, dust, snow etc.), WTs (Wind Turbines) undergo constantly changing loads, which result in highly variable operational conditions that lead to intense mechanical stress [1]. Consequently, the operational unavailability of WTs reaches $3 \%$ of the lifetime of a WT. Moreover, operation and maintenance costs can account for $10 \%-20 \%$ of the total cost of energy for a wind project [2], and this percentage will be much higher for a WT at the end of life. This high cost hinders the wind power's more rapid development.

Condition monitoring plays an important role in the maintenance and repairing [3], which could provide first indication evidence that shows something is wrong [4]. Traditional condition monitoring approaches focus on the expensive components (such as bearing, generator, gear boxes, etc.) fault detection [5-7], such as vibration analysis and oil analysis [8]. But this method must use an independent system, which needs to install additional sensors and hardware that is pretty costly.

Detecting WTs' faults and operation problems based on SCADA system data is a very cost-effective way [9] and WT's power performance character is often selected to be used to monitor WT's overall health. In this method, building an available power curve model is critical important.

Project supported by the National Natural Science Foundation of China(No. 651277023) and Jilin Province Science and Technology Development Project(20150204084GX). 
Zaher [10] proposed to obtain the power curve model with figuring out the inner and outer alarm limits of wind-power generation model by selecting a number of healthy turbines. Shokrzadeh [11] focused on power curve modeling based on available operational output power data using four parametric and nonparametric methods. Kusiak, A et al. developed the RBF neural network and MLP neural network to obtain the optimum power curve model [12]. Huan Long [13] proposed the evolutionary algorithms (EA), ant colony algorithm (PSO), and artificial immune network (aiNet) to get the power curve model. Philip Cross proposed power curve models that were respectively established with linear, state dependent parameter models, artificial neural network (ANN) [14]. Kusiak, A et al. respectively investigated the least square method, maximum likelihood estimation method and k-NN algorithm to construct power curve model [15].

One common limitation of above algorithms is that they should include abnormal data as less as possible so as to obtain satisfactory power curve model. However, it is not conform to the actual situation in many cases. Moreover, in order to adapt to the changes of the seasons and the wind turbine self-condition, the model needs to be updated frequently, but most of above algorithms require a great computational overhead training process to build the power curve model, which result in low efficiency and great difficulty to apply in real-time monitoring.

In this paper, the impact of WT's abnormal data on power curve is studied and new algorithms were developed to ensure the power curve model could be used in actual field application. Betz' law and RC model are new constraints that are developed to reject extreme abnormal data so as to govern the power curve model. The Inner-DBSCAN algorithm is proposed for further rejecting abnormal data. Consequently, normal data is obtained, and edge recognition is presented to build power curve model with the normal data. The proposed algorithm automatically generates an alarm message when the windpower data measured at the wind turbine deviate from the limits of power curve model. In addition, the developed power curve model based condition monitoring was actually applied on eight blind industrial studies, and the results shows its effectiveness and great advantages.

\section{The Impact of Abnormal Data on Power Curve}

The power performance of wind turbine is represented by the wind-power $(\mathrm{P}-\mathrm{V})$ curve. According to the energy conservation law and Betz' law discussed in [16], the real active power is given by

$$
P=0.5 \rho \pi R^{2} C_{p} v^{3}
$$

where $\mathrm{P}$ is theoretical power captured by the rotor of a wind turbine, $\rho$ is the air density, $\mathrm{R}$ is the radius of the rotor determining its sweeping area, $\mathrm{V}$ is the wind speed, $\mathrm{Cp}$ is the power coefficient.

Although WT's power curve model is clear by equation (1), its capability of providing accurate prediction of generated wind power is limited due to its inherent disadvantages. Firstly, in the WT design, the air density is assumed to be constant. Secondly, prediction error or design parameter error will magnify in $\mathrm{v}^{3}$ [16].

Consequently, the power performance of the normal operation of each turbine is not the same and the direct use of power curve provided by WT manufacturers to monitor of abnormal operation status is inappropriate. It is need to carry out specific WT's power curve analysis based on actual operating data.

WTs' abnormal data has a terrible impact on power curve. In the essence, abnormal data is generated by shutting down, downrating and overating result of WTs' failures, lack of maintenance and sensors' faults. In addition, abnormal data sometimes is generated result of limiting power by the power dispatching agencies. In order to analyze the power performance of WTs, SCADA datasets is collected for 
analyzing. Figure 1 shows one WT' wind speed and power sequence diagram from 4:48 one day to $1: 00$ the next day with a $10 \mathrm{~min}$ interval. The cut-in wind speed $\mathrm{v}_{\mathrm{i}}=$ $2.5 \mathrm{~m} / \mathrm{s}$, rated wind speed $\mathrm{v}_{\mathrm{r}}=11 \mathrm{~m} / \mathrm{s}$, cut-out wind speed $\mathrm{v}_{\mathrm{o}}=20 \mathrm{~m} / \mathrm{s}$ and the WT rated at $1500 \mathrm{KW}$. When $4: 48<\mathrm{t}<6: 32$, the wind speed $\mathrm{v}_{\mathrm{i}}<\mathrm{v}<\mathrm{v}_{\mathrm{o}}, \mathrm{p}=0$, at this period the WT is with halt status. When 8:12<t<7:52, 8:56 $<\mathrm{t}<9: 30$ and 10:16 $<\mathrm{t}$ $<16: 48$, the average wind speed in these three time periods is about $8 \mathrm{~m} / \mathrm{s}$. According to the manufacturer's power curve, the output power should reach $700 \mathrm{KW}$, but the actual output of the WT was limited to about $250 \mathrm{KW}$. It's clearly that the WT runs at a lower generation status in this three periods.

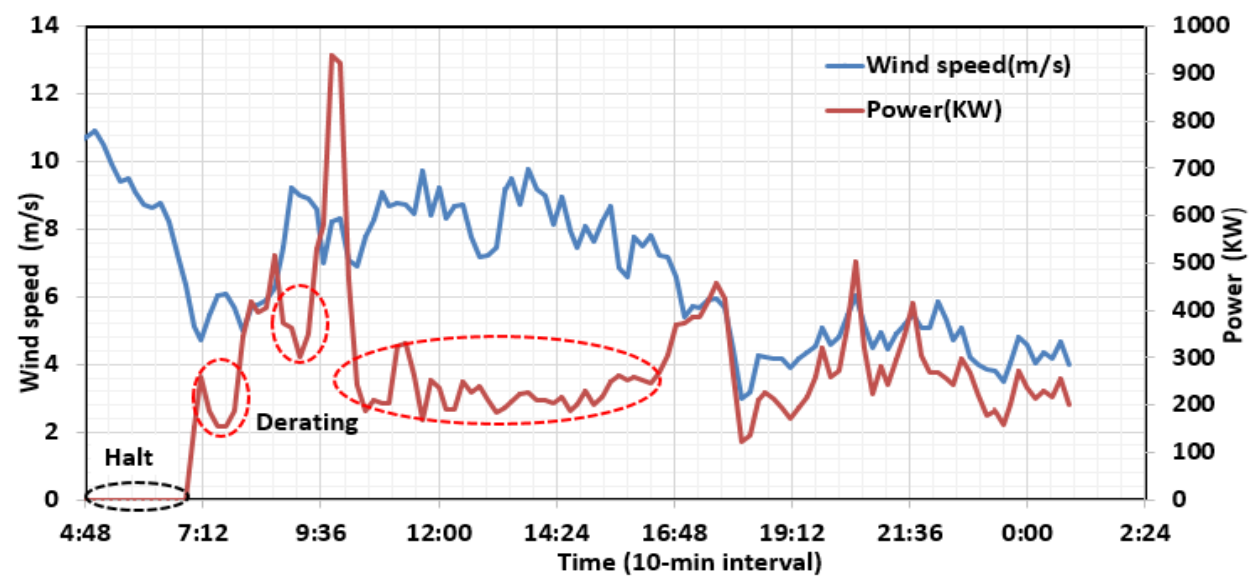

Figure 1. Wind Speed and Power Sequence Diagram

In order to get abnormal data's further impact on power curve, as seen in Figure 2 shows, one month's wind speed and power data is collected and the datasets are expressed with $U$.

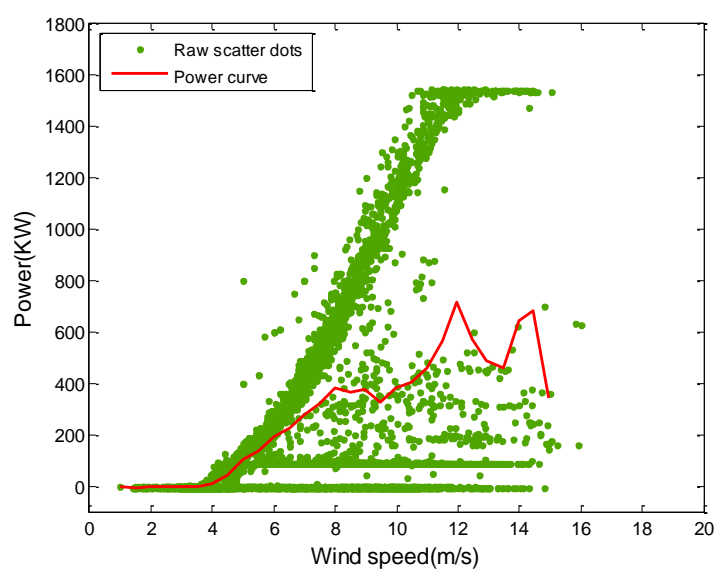

Figure 2. Power vs Wind Speed Scatter Dots

Clearly, all kinds of data, includes normal operation data, abnormal operation data, halt data and data with random error or system error, are mixed together so that the power curve is distorted sharply. It's obviously known that the measured power curve could not be seen as a reference of WTs' power performance. 


\section{Power Curve Model}

\subsection{Data Preprocessing}

Extreme abnormal data has big impact on modeling power curve, so it's essential to take procedures to reject this kind of data. In the following, data preprocessing is done with the power optimization constraint and power lowest constraint, which could be used to reject the extremely high and low power generation data.

As for power optimization constraint, it is computed based on Betz' law. WT power coefficient can be computed as [17]:

$$
C_{p}=\frac{P}{\frac{1}{2} \rho S v^{3}}
$$

where $\rho$ is the air density, $S$ is the WT wind rotor's swept area, $v$ is the wind speed, $P$ is the WT's generated power. According to Betz' law, the maximum power coefficient is 0.593. Based on this law, the upper boundary (we called Betz Boundary) of the power curve model is established.

As for power lowest constraint, it is computed with power generation model (we named RC model) that proposed by R.Chedid [18]. RC model is showed as follows:

$$
\begin{cases}P W=0 & v<v_{c i} \\ P W=a \cdot v^{3}-b \cdot P_{r} & v_{c i}<v<v_{r} \\ P W=P_{r} & v_{\mathrm{r}}<v<v_{\mathrm{co}} \\ P W=0 & v>v_{c o}\end{cases}
$$

where $\mathrm{a}=\frac{P_{\mathrm{r}}}{v_{r}{ }^{3}-v_{c i}{ }^{3}}, b=\frac{v_{c i}{ }^{3}}{v_{r}{ }^{3}-v_{c i}{ }^{3}}$, and $P_{r}, \quad V_{c i}, \quad V_{r}, \quad V_{c o}$ are the rated power, cut-in ,rated and cut out wind speeds respectively.

$P=P W \times A w \times e f f_{w}$

where $A_{w}$ is the swept area of the wind wheel, eff $w$ is the WT's energy utilization efficiency. For getting an available WT power lowest constraints, an extremely low value 0.05 is assigned to eff $f_{w}$ and the lower boundary (we called RC Boundary) is accordingly obtained.

Consequently, extreme abnormal operating datasets are filtered out by Betz Boundary and RC Boundary as Figure 3 shows. As a result, normal data sets $U_{\text {pre-normal }}$ can be obtained after data preprocessing. 


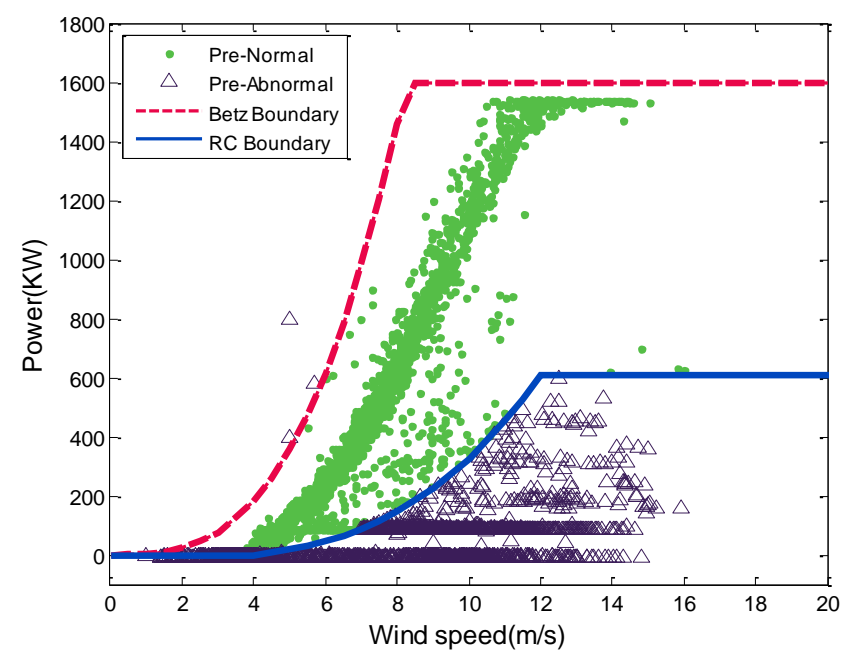

Figure 3. Data Preprocessing by Betz Boundary and RC Boundary

\subsection{Data Clustering with Inner-DBSCAN Algorithm}

Data clustering with Inner-DBSCAN algorithm is the core procedure of abnormal data rejection, which could clustering most abnormal data.

\subsubsection{DBSCAN Algorithm}

DBSCAN (Density-based spatial clustering of applications with noise) algorithm was proposed by Martin Ester in 1996, it is famous for density-based clustering. It has the great advantage of identifying any shape clusters and isolated points, which is consistent with the distribution of wind speed-power scatter dots [19]. DBSCAN identifies a cluster with given density thresholds, which are determined by parameters Eps and Minpts (where Eps represents the radius, Minpts represents the least number of core points within the Eps radius).

Parameter Eps of the DBSCAN algorithm is calculated by classical Euclidean distance as [19]:

$d_{i, j}=\left|x_{i}-x_{j}\right|$

where $x_{i}$ represents the $\mathrm{i}$-th dot, $x_{j}$ represents the $\mathrm{j}$-th dot.

Cluster center is defined as the mean value of the elements in the cluster, and is presented by

$w_{j}=\frac{1}{m} \sum_{i=1}^{m} x_{i}$

where $m$ is the object number that the $j$-th cluster contains.

Four statistical metrics is discussed in [16] were used to evaluate the obtained windpower model:

$M A E=\frac{1}{n} \sum_{i=1}^{n}\left|\hat{y}_{i}-y_{i}\right|$

$\operatorname{SDofAE}=\sqrt{\frac{1}{n} \sum_{i=1}^{n}\left(\left|\hat{y}_{i}-y_{i}\right|-\frac{1}{n} \sum_{i=1}^{n}\left|\hat{y}_{i}-y_{i}\right|\right)^{2}}$ 


$$
\text { MAPE }=\frac{1}{n} \sum_{i=1}^{n}\left|\frac{\hat{y}_{i}-y_{i}}{y_{i}}\right| \times 100 \%
$$

$$
\text { SDofAPE }=\sqrt{\frac{1}{n} \sum_{i=1}^{n}\left(\left|\frac{\hat{y}_{i}-y_{i}}{y_{i}}\right|-\frac{1}{n} \sum_{i=1}^{n}\left|\frac{\hat{y}_{i}-y_{i}}{y_{i}}\right|\right)^{2} \times 100 \%}
$$

DBSCAN has an obvious disadvantage that when data distribution is uneven, the clustering result is bad due to the use of a unified global variables. As datasets $U_{\text {prenormal }}$ for example, the clustering result as Figure 4 shows $($ Minpts $=5, E p s=0.3271)$. The clustering result is terrible.

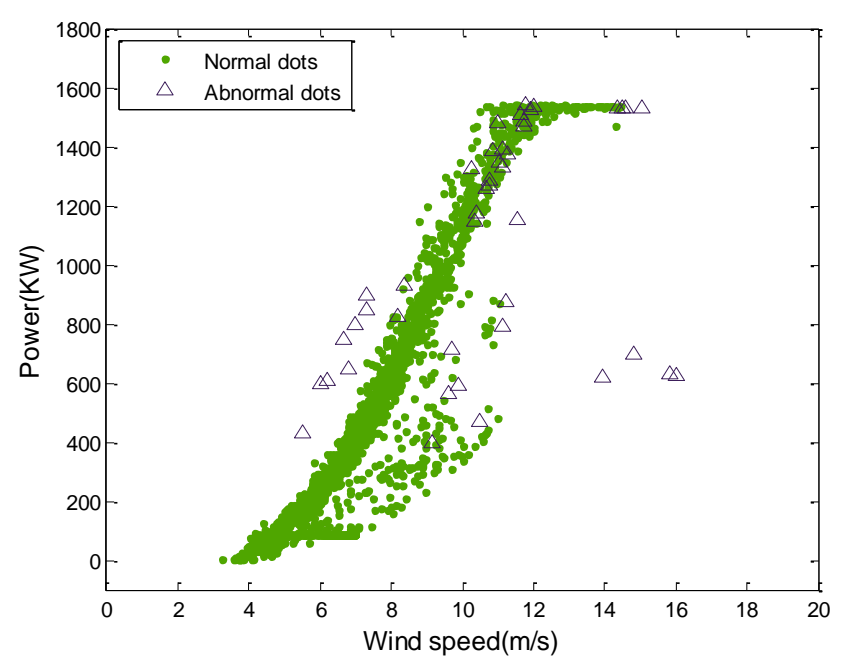

Figure 4. DBSCAN Clustering Result

Based on the distribution characteristic of power-wind speed scatter dots, this paper proposed an interval based DBSCAN algorithm (Inner-DBSCAN) in the following.

\subsubsection{Inner-DBSCAN Algorithm}

The datasets $U_{\text {pre-normal }}$ shall be separated in equal intervals and in every interval the DBSCAN algorithm is used.

Firstly, the datasets is classified according to $0.5 \mathrm{~m} / \mathrm{s}$ interval of the wind speed. Each interval center is an integer multiple of $0.5 \mathrm{~m} / \mathrm{s}$, and data set $U_{i}$ is assumed to obtain in each interval. $v_{\max }$ is supposed the highest wind speed, then the biggest interval will be $\mathrm{n}=\left[v_{\max } / 0.5\right]$. Datasets of each interval is defined as follows:

$$
U_{i}=\{(v, p) \mid(v, p) \in U, 0.5 i-0.25<v<0.5 i+0.25\}, i \in(0,1, \ldots, n)
$$

where $(v, p)$ is element in the i-th interval.

Secondly, do data clustering in each interval by DBSCAN algorithm. DBSCAN algorithm based on the fact that a cluster can be uniquely identified by any of these core objects, that is for any data point $\mathrm{p}$ meets core point condition, all points from $\mathrm{p}$ that is density-reachable [19] in database D consist of a complete sets of cluster and $p$ belongs to C. Datasets that are not included in any cluster constitute noise points.

Finally, identify normal cluster and re-clustering the data. In the previous process, the extreme abnormal scatter points on the top left has been removed, so the final desired normal data retention must have been gathered in the upper part and the left part of the scatterplot, so the larger mean value cluster should be retained. Using secondary clustering fusion theory: (1) In above intervals, clustering result is $\left\{C_{l}, C_{2}, \ldots, C_{m}\right\}$ and 
sometimes there is also a noise cluster, and calculate the clustering center of $\mathrm{m}$ clusters $\left\{w_{1}, w_{2}, \ldots, w_{m}\right\}$; (2)find out the maximum value in $\left\{w_{1}, w_{2}, \ldots, w_{m}\right\}$ and accordingly $C_{\max }$ is determined as normal cluster $C_{\text {pre-normal }}$ preliminarily; (3) calculate the distance $\mathrm{d}_{\mathrm{i}}$ between $w_{\max }$ and $w_{i}$ (not $w_{\max }$ ), and $d_{i}=w_{\max }-w_{i}$; (4)compare each $\mathrm{d}_{\mathrm{i}}$ with preset value $T$ (as $0.2 P_{r}$ ). And if $d_{i}<T$, then $C_{i}$ is determined as normal cluster and merge $C_{\max }$ with $C_{i}$, otherwise $C_{i}$ is determined as abnormal cluster.

Figure 5 shows four randomly selected intervals clustering results with datasets $U_{\text {pre- }}$ normal by Inner-DBSCAN algorithm. Obviously, the clustering result is fantastic in each interval.

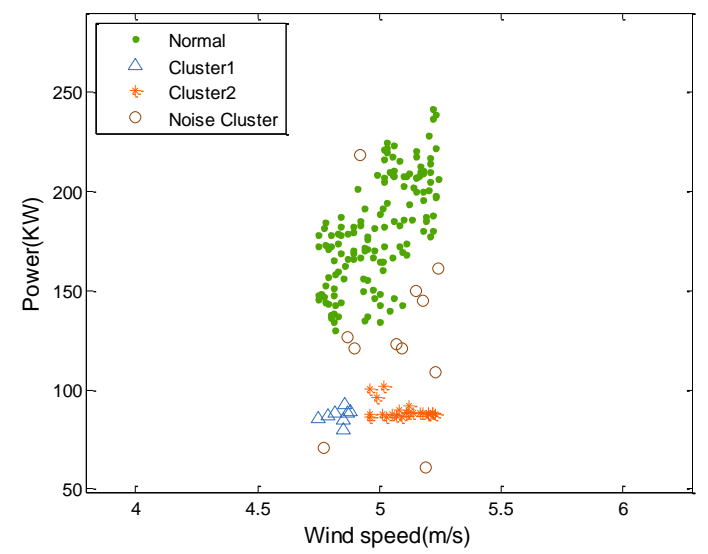

(a)

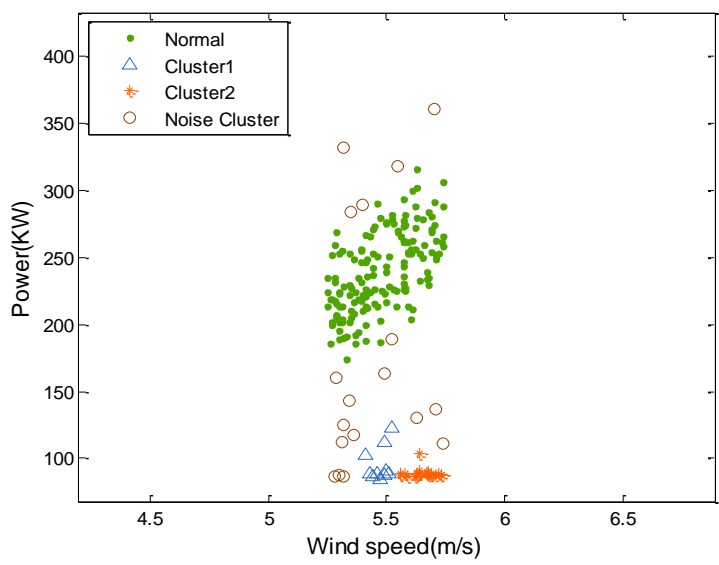

(b) 


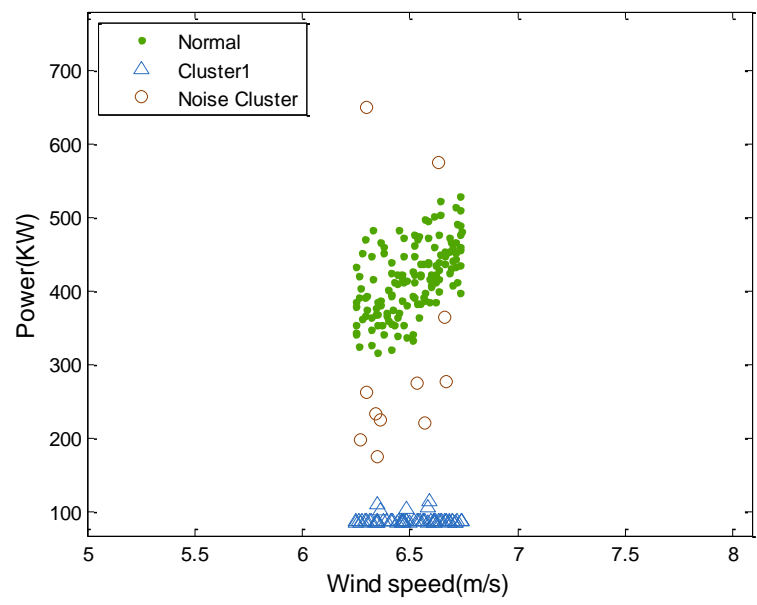

(c)

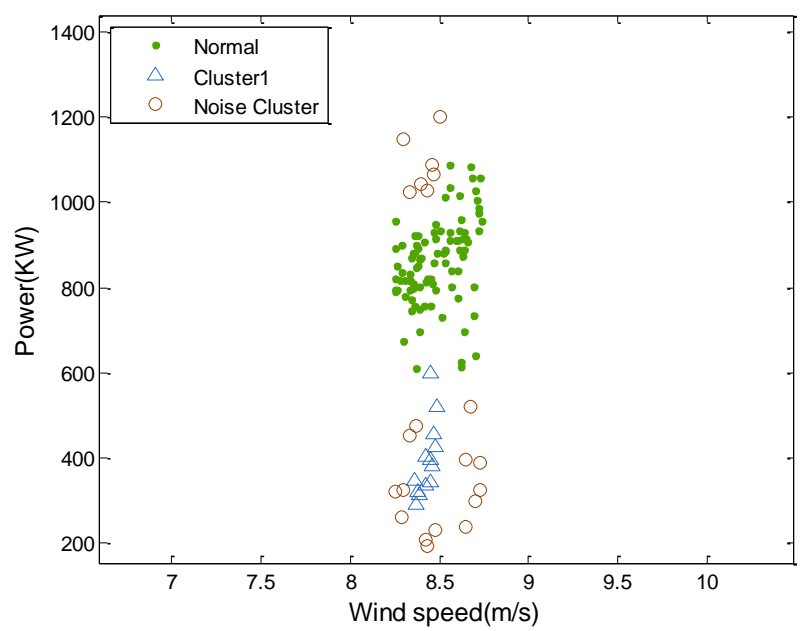

(d)

Figure 5. Inner-DBSCAN Algorithm Clustering Results in Random Four Intervals

Put the clustering results of each interval together, and overall clustering result is obtained as Figure 6 shows. Obviously, the clustering result is much better than pure DBSCAN, and the vast majority of the abnormal status can accurately be identified. But there are some normal points that are mistaken for abnormal $(8.5 \mathrm{~m} / \mathrm{s}$ or so and $13 \mathrm{~m} / \mathrm{s}$ or so), and vise versa in some case. To solve above problems, an effective additional step by the power curve model method is presented in the following. 


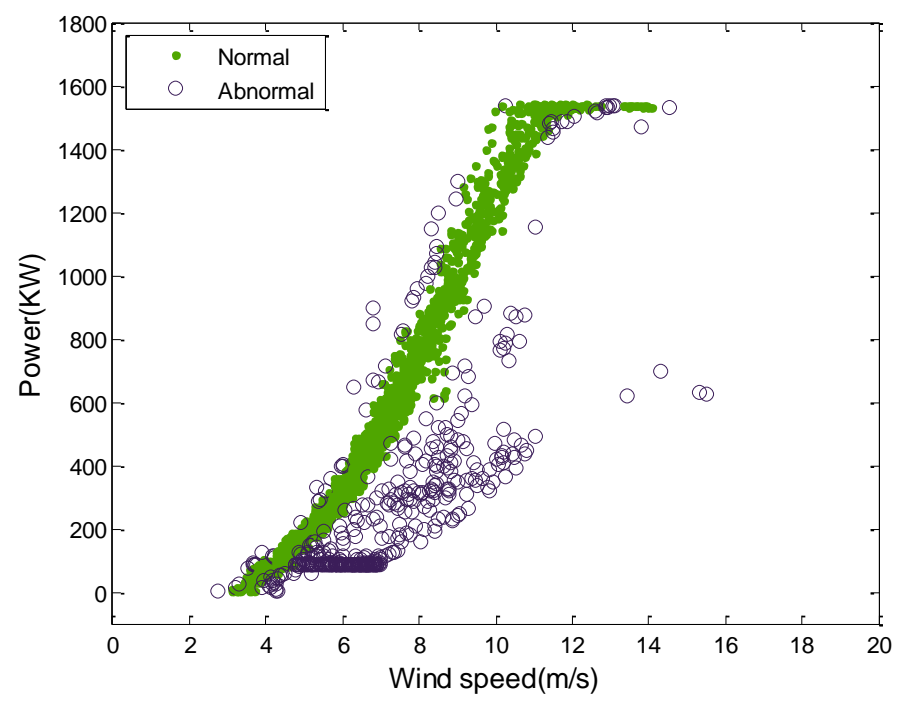

Figure 6. Inner-DBSCAN Algorithm

\subsection{Power Curve Model}

Power curve model is established by the recognition of the edge of normal data. Figure out the maximum and minimum power values in each interval's mean wind speed neighborhood and the most values serve as the points at upper and lower boundaries of the power curve model. The mean wind speed value $v_{\text {mean }}$ is assumed in the $\mathrm{i}$-th interval with a smaller neighbourhood radius $\delta$ is given and dataset $\mathrm{Q}_{\mathrm{i}}$ is supposed in $\left[v_{\text {mean }}-\delta, v_{\text {mean }}+\delta\right]$.Figure out the maximum power $p_{i, \text { max }}$ and minimum power $p_{i, \text { min }}$. Then the point $\left(v_{\text {mean }}, p_{i, \max }\right)$ and the point $\left(v_{\text {mean }}, p_{i, \text { min }}\right)$ are seen as the points on the upper and lower boundary of the i-th interval. Then the power curve model is obtained by $n$ couple of boundary points in $n$ intervals as Figure 7 shows.

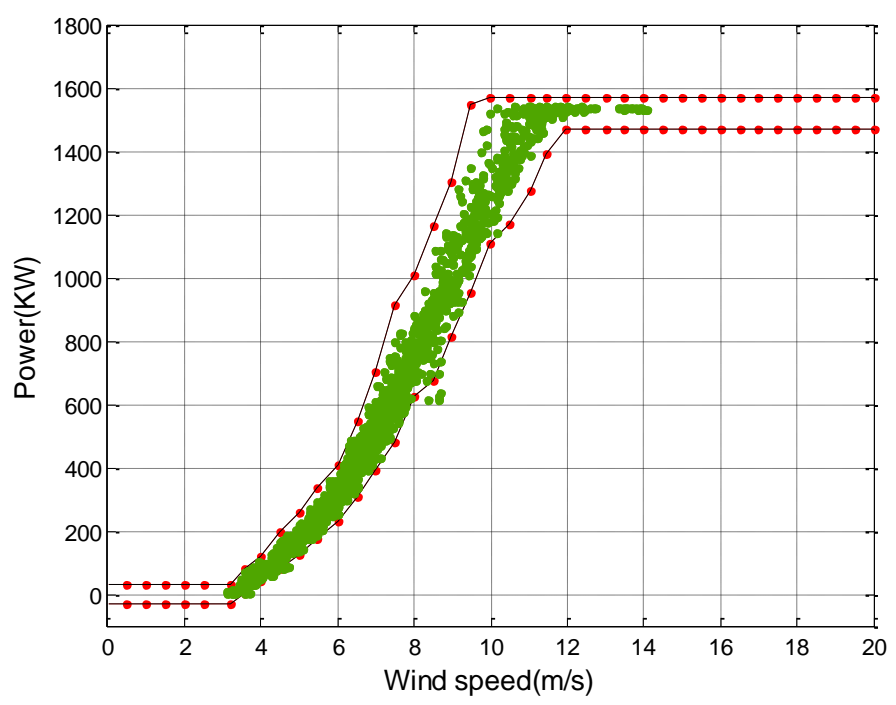

Figure 7. Power Curve Model 


\section{Anomaly Detection}

WT operation status at $t_{j}$ time is determined by judging if $m\left(v_{j}, p_{j}\right)$ is within normal limits by power curve model and linear interpolation method.

Linear interpolation algorithm is calculated by

$k_{i}=\frac{p_{i+1}-p_{i}}{v_{i+1}-v_{i}}$

$b_{i}=p_{i}-k_{i} \cdot v_{i}$

For any dataset $\left(v_{j}, p_{j}\right), v_{i}<=v_{j}<v_{i+1}$, upper boundary slope $k_{\text {highest }, i}$ and offset $b_{\text {highest }, i}$ is calculated and then the maximum power $p_{\text {highest }}$ is obtained by

$$
p_{\text {highest }}=\mathrm{k}_{\text {highest }, i} \cdot v_{j}+b_{\text {highest }, i}
$$

The minimum power $p_{\text {lowest }}$ could also be calculated as the same. If $p_{\text {lowest }}<=p_{j}<=p_{\text {highest }}$ then $m\left(v_{j}, p_{j}\right)$ is determined as normal operation status, otherwise is determined as abnormal operation status. Consequently, operation status of the initial datasets $U$ could be determined by the power curve model as Figure 8 shows.

Expected power could be obtained by power curve that based on normal data. Then the residual power could be calculated between measured and expected power. Figure 9 shows the sequence chart of residual power.

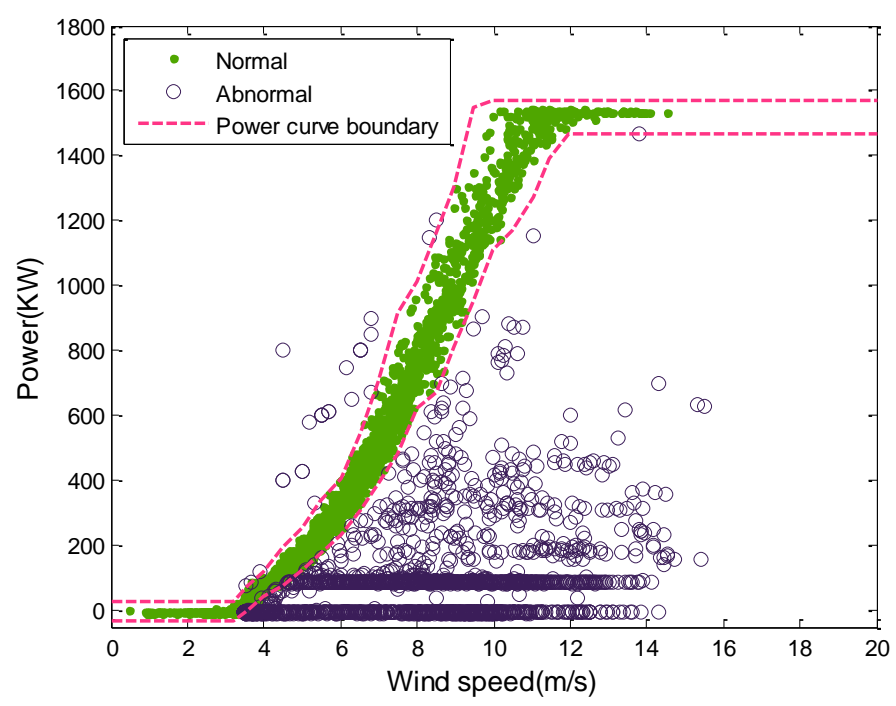

Figure 8. Re-Cluster by Power Curve Model 


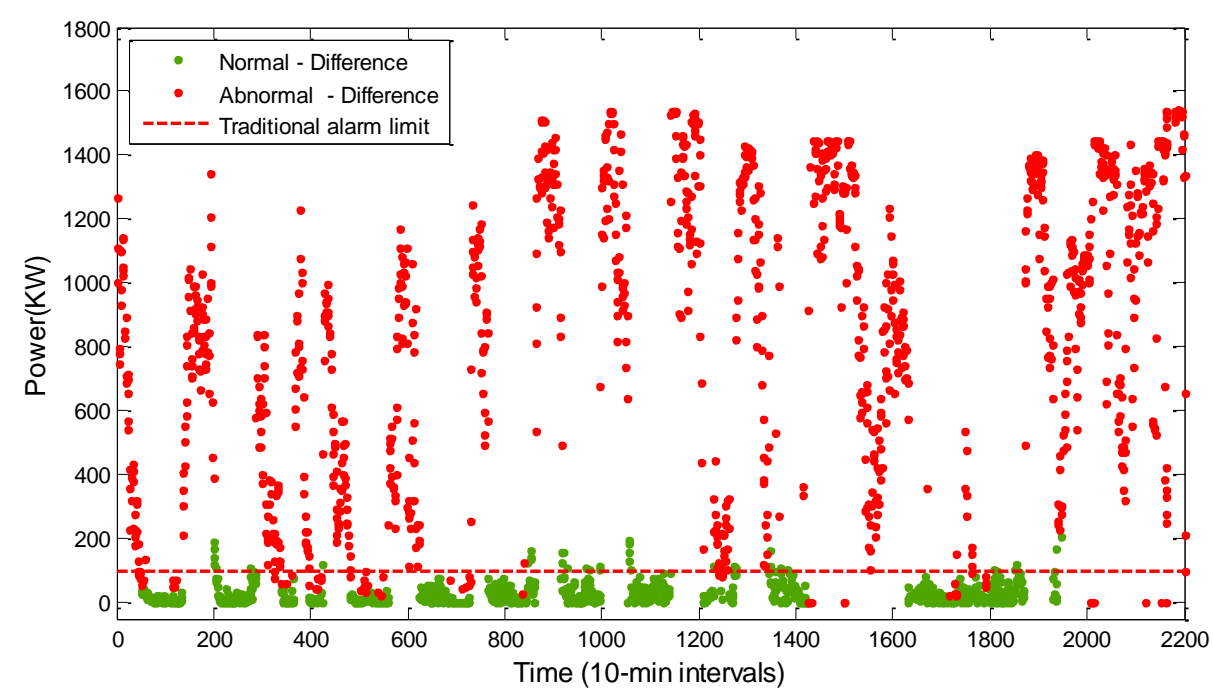

Figure 9. Sequence Chart of Residual Power

An alarm limit is set with usual method as Figure 9 shows, with a result that some normal data is mistook for abnormal or some abnormal data is mistook for normal. The power curve model method presented in this paper could adjust the alarm limits automatically. As a result, anomaly could be effectively and accurately monitored. But this could result in much too many alarms. The method to solve this problem is that setting an appropriate coefficient for upper and lower boundaries to magnify the normal data range.

\section{Industrial Case Studies}

In order to verify the effectiveness of the proposed algorithm, we use SCADA data of 8 wind turbines from different wind farms to do analysis. Figure 10 shows the clustering results of three random selected WTs' data which come respectively from WT3\# in January, WT12\# in May and WT16\# in May.

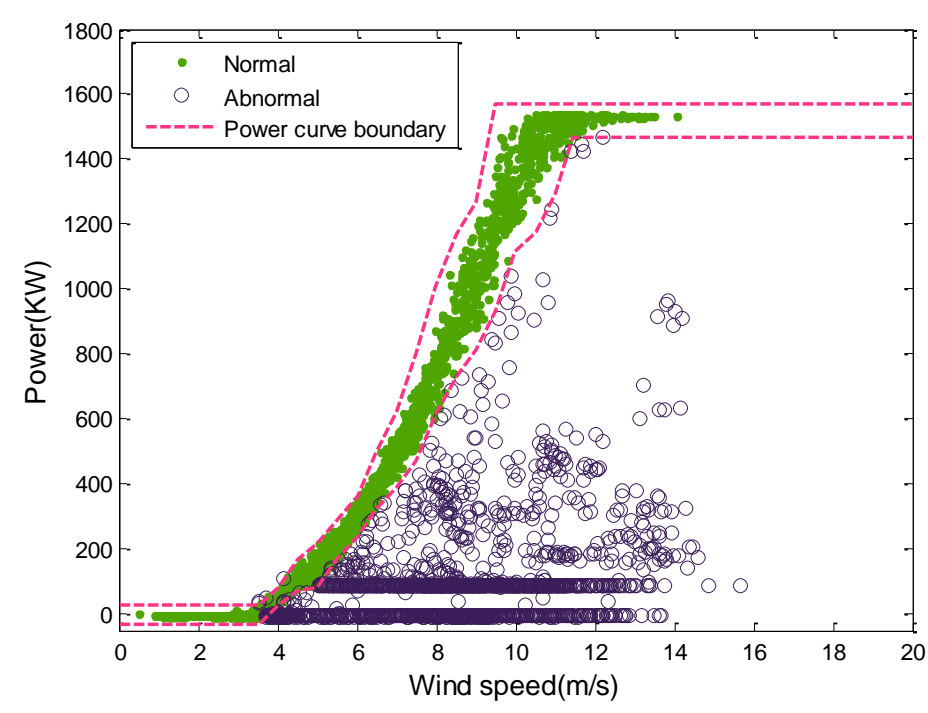

(a) 


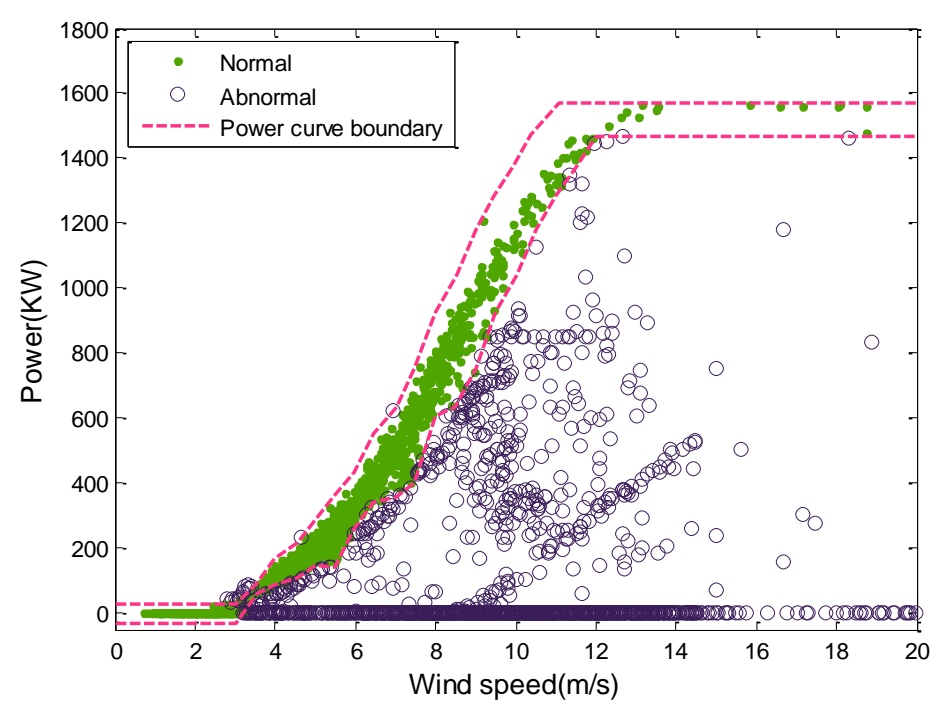

(b)

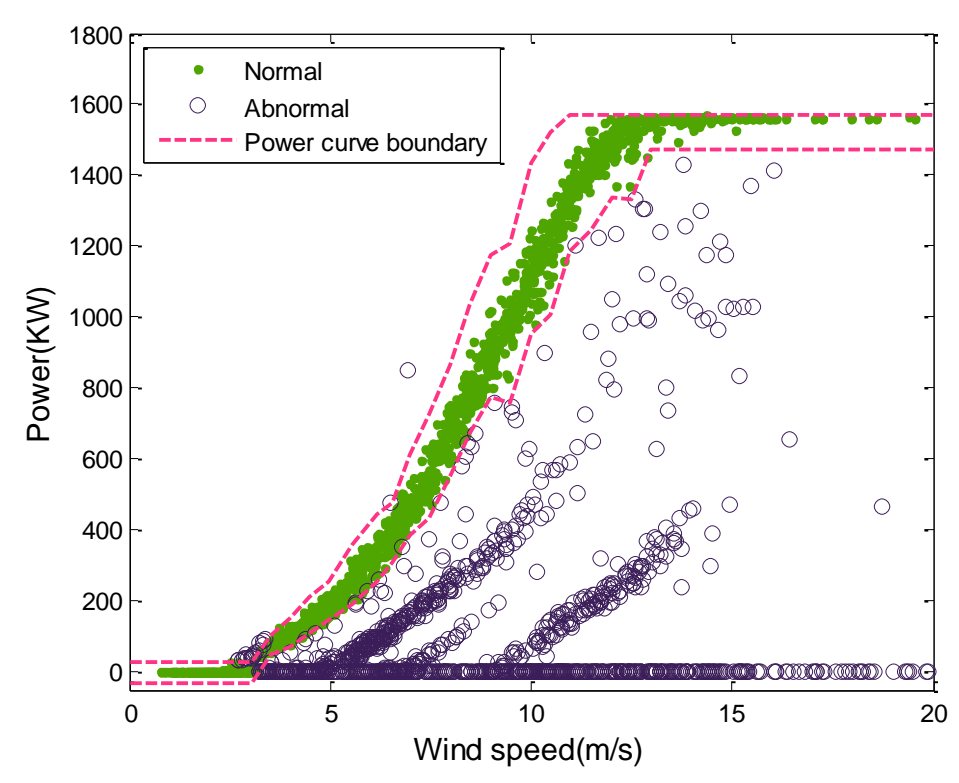

(c)

Figure 10. Inner-DBSCAN Based Clustering Results

Obviously, the method presented in this paper can detect the abnormal operation status. There may be many reasons for the anomaly in above three pictures, like sensor faults, pitch angle control failure, inappropriate blade angle setting, blade damage, control program error, operation with limited power, environmental conditions (adheres to insects, dirt attached, blade icing) etc. .

The results of clustering statistical analysis of three wind turbines are shown in Table1. Raw refers to the analysis results based on the raw data, Betz\&RC refers to the analysis results of the datasets $U_{\text {pre-normal }}$ that after data preprocessing, DBSCAN refers to the analysis results with the classical DBSCAN algorithm on the basis of the datasets $U_{\text {pre }}$ normal, Inner-DBSCAN refers to the analysis results obtained with the Inner-DBSCAN algorithm that this paper presented based on datasets $U_{\text {pre-normal }}$.

Obviously, as a whole, it seems that Raw has the maximum value of four kinds of clustering statistical analysis index, which shows the large dispersion. The dispersion has 
been significantly reduced after Betz \& RC process. The effect of the classical DBSCAN algorithm on the reduction of the index is very small, but the Inner-DBSCAN algorithm can effectively reduce the index. MAE index of the three wind turbines was reduced by an average of $(11 \%)$ by DBSCAN, whereas reduced $60 \%$ by Inner-DBSCAN; as well, the MAE index of the three wind turbines was reduced by an average of (2\%) by DBSCAN, whereas reduced $62 \%$ by Inner-DBSCAN. From the clustering statistical analysis index and the clustering results in Figure 10, it is clear that the method presented in this paper can effectively do WTs' condition monitoring.

Table 1. Statistical Metrics of 4 Conditions

\begin{tabular}{|l|l|l|l|l|}
\hline SM & Raw & $\begin{array}{c}\text { Betz \& } \\
\text { RC }\end{array}$ & DBSCAN & $\begin{array}{c}\text { Inner- } \\
\text { DBSCAN }\end{array}$ \\
\hline MAE1 & 239.50 & 129.51 & 115.11 & 60.31 \\
\hline SofAE1 & 213.35 & 135.03 & 119.13 & 55.51 \\
\hline MAPE1 & 204.08 & 112.65 & 111.83 & 67.04 \\
\hline SofAPE & 138.55 & 117.27 & 118.46 & 114.99 \\
\hline MAE2 & 135.82 & 86.40 & 75.74 & 29.20 \\
\hline SofAE2 & 180.14 & 105.64 & 94.32 & 28.91 \\
\hline MAPE2 & 172.19 & 24.73 & 24.26 & 10.44 \\
\hline SofAPE & 171.80 & 6.30 & 6.55 & 2.70 \\
\hline MAE3 & 238.03 & 108.72 & 99.21 & 26.20 \\
\hline SofAE3 & 221.64 & 118.50 & 113.70 & 25.93 \\
\hline MAPE3 & 354.40 & 45.38 & 49.06 & 5.81 \\
\hline SofAPE & 518.23 & 33.92 & 36.26 & 1.05 \\
\hline
\end{tabular}

The total algorithm is programmed with MATALB, and runs in the ordinary PC with each WT's single month' data from the eight wind turbines respectively be processed. And a large number of experiments show that the clustering result is the best when single month data is processed, where too much data will cause the clustering boundary fuzzy and too little data will result in too much clusters. Clustering execution time is shown in Table2, the average processing time is $1.08 \mathrm{~s}$, and it will be much faster when the program runs on a higher configuration machine, and real-time application is practicable.

Table 2. Execution Time Statistics of Inner-DBSCAN Algorithm

\begin{tabular}{|c|l|}
\hline $\begin{array}{c}\text { Unit } \\
\text { number }\end{array}$ & Execution time /s \\
\hline$\# 3$ & 1.14 \\
\hline$\# 5$ & 1.05 \\
\hline$\# 8$ & 0.94 \\
\hline$\# 9$ & 1.16 \\
\hline$\# 10$ & 0.99 \\
\hline$\# 11$ & 1.07 \\
\hline$\# 12$ & 1.06 \\
\hline$\# 16$ & 1.23 \\
\hline
\end{tabular}

\section{Conclusion}

A data-driven method based on power curve model was proposed for condition monitoring for wind turbines in this study. Data preprocessing based on Betz' law 
and RC model greatly improve the effect of the data clustering and plays an important supervisory role for building the power curve model. Furthermore, taking advantage of DBSCAN algorithm that clustering based on density and considering the distribution characteristic of wind-power scatter dots, we proposed the InnerDBSCAN algorithm, and got a pretty good clustering result for power curve model.

Maximum and minimum of interval mean wind speed neighborhood method was effectively used to identify the edges of the normal operation data. As a result, a power curve model is formed based on the edges. With the power curve model, abnormal operation condition can be effectively monitored.

The condition monitoring method for WTs in this paper was investigated based on eight blind cases. The results indicated that this method is with high universality and small computational overhead. The method has a high application value in condition monitoring for wind turbines.

\section{Acknowledgments}

The authors wish to thank Long Yuan (Beijing) Wind Power Engineering Technology CO., LTD for providing the industrial experiments. And thank Jie Cao for her help in English proofreading of the manuscript.

\section{References}

[1] P. Tchakoua, R. Wamkeue and M. Ouhrouche, "Wind turbine condition monitoring: State-of-the-art review, new trends, and future challenges", Energies, vol. 7, no. 4, (2014), pp. 2595-2630.

[2] C.A. Walford, "Wind turbine reliability: understanding and minimizing wind turbine operation and maintenance costs", Department of Energy of USA, (2006).

[3] Z. Hameed, Y.S. Hong and Y.M. Cho, "Condition monitoring and fault detection of wind turbines and related algorithms: A review", Renewable and Sustainable energy reviews, vol. 13, no. 1, (2009), pp. 139.

[4] J.Y. Park, J.K. Lee and K.Y. Oh, "Development of a novel power curve monitoring method for wind turbines and its field tests", Energy Conversion, IEEE Transactions on, vol. 29, no. 1, (2014), pp. 119128.

[5] C.J. Crabtree, D. Zappalá and P.J. Tavner, "Survey of commercially available condition monitoring systems for wind turbines", (2014).

[6] S. Sheng, F. Oyague and S. Butterfield, "Investigation of various wind turbine condition monitoring techniques", 7th International Workshop on Structural Health Monitoring, Stanford, USA, September 13 (2009).

[7] E. Wiggelinkhuizen, T. Verbruggen and H. Braam, "Assessment of condition monitoring techniques for offshore wind farms", Journal of Solar Energy Engineering, vol. 130, no. 3, (2008), pp.1004-1112.

[8] Y. Amirat, M.E.H. Benbouzid, B. Bensaker and R. Wamkeue, "Condition monitoring and fault diagnosis in wind energy conversion systems: a review", Electric Machines and Drives Conference, Antalya, Turkey, May 3-5 (2007).

[9] K. Kim, G. Parthasarathy, O. Uluyol and W. Foslien, "Use of SCADA data for failure detection in wind turbines", ASME 2011 5th International Conference on Energy Sustainability, Washington, USA, August 7-10 (2011)

[10] A.S. Zaher and S.D.J. McArthur, "A multi-agent fault detection system for wind turbine defect recognition and diagnosis", Power Tech 2007, Lausanne, Switzerland, July 1-5 (2007).

[11] S. Shokrzadeh, M.J. Jozani and E. Bibeau, "Wind Turbine Power Curve Modeling Using Advanced Parametric and Nonparametric Methods", IEEE T Sustainable Energy, vol. 5, no. 4, (2014), pp. 1262 1269.

[12] A. Kusiak, H. Zheng and Z. Song, "Power optimization of wind turbines with data mining and evolutionary computation", Renewable energy, vol. 35, no. 3, (2010), pp. 695-702.

[13] H. Long, L. Wang and Z. Zhang, "Data-Driven Wind Turbine Power Generation Performance Monitoring”, Industrial Electronics, IEEE Transactions on, vol. 62, no. 10, (2015), pp. 6627-6635.

[14] P. Cross and X. Ma, "Model-based condition monitoring for wind turbines", Proceedings of the 19th International Conference on Automatic Computing, San Jose, USA, June 1-7 (2013).

[15] A. Kusiak, H. Zheng and Z. Song, "On-line monitoring of power curves. Renewable Energy", vol. 34, no. 6, (2009), pp. 1487-1493.

[16] Z. Zhang, Q. Zhou and A. Kusiak, "Optimization of wind power and its variability with a computational intelligence approach”, IEEE T Sustainable Energy, vol. 5, no. 1, (2014), pp. 228-236. 
[17] "Power performance of electricity producing wind turbines based on nacelle anemometry", International Electrotechnical Commission, IEC 61400-12-2 CD Part 12-2, (2008).

[18] R. Chedid, H. Akiki and S. Rahman, "A decision support technique for the design of hybrid solar-wind power systems", IEEE T Energy conversion, vol. 13, no. 1, (1998), pp. 76-83.

[19] M. Ester, H.P. Kriegel, J. Sander and X. Xu, "A density-based algorithm for discovering clusters in large spatial databases with noise", vol. 96, no. 34, (1996), pp. 226-231.

\section{Authors}
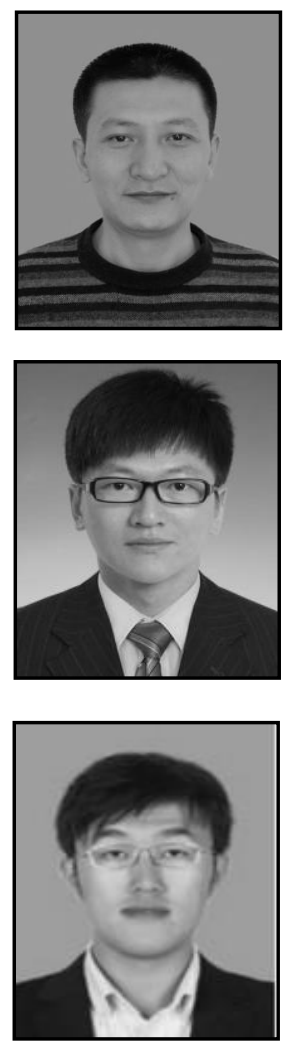

Jianlou Lou, He is currently a associate professor in the School of Information Engineering at Northeast DianLi University, Jilin, China. His research interests include big data analysis, data mining and smart power grids.

Kai Shan, He is currently pursuing his M.S. degree in the School of Information and Engineering at Northeast DianLi University, Jilin, China.

His research interests include wind turbine data analysis \& data mining, wind turbine monitoring as well as wind turbine power performance test.

Jia Xu, He received the M.S. degree in Instrument Science and Technology at Xi'an Jiaotong University, China, in 2010.

Now, he is the head of the Center of the Wind Data Analysis and Performance Optimization at LongYuan (Beijing) Wind Power Engineering Technology CO., LTD., Beijing, China. 
International Journal of Control and Automation Vol. 9, No. 3 (2016) 